\title{
The effect of vacuum annealing on the structure and properties of the electrically conductive a-CN coating
}

\author{
A. Poplavsky ${ }^{\text {a,b }}$, Yu Kudriavtsev ${ }^{\text {c, }}$, A. Kolpakov ${ }^{\text {a }}$, E. Pilyuk ${ }^{\text {a }}$, S. Manokhin ${ }^{\text {d }}$ I. Goncharov ${ }^{\text {a,b }}$

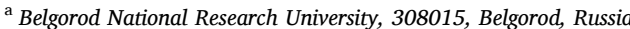 \\ ${ }^{\mathrm{b}}$ Belgorod State Technological University Named After V.G. Shoukhov, 308012, Belgorod, Russia \\ ${ }^{\mathrm{c}}$ Departamento Ingeniería Electrica, SEES, CINVESTAV, 07360, Mexico \\ ${ }^{\mathrm{d}}$ Institute of Problems of Chemical Physics, Russian Academy of Sciences, 142432, Chernogolovka, Russia
}

\section{A R T I C L E I N F O}

\section{Keywords:}

Nitrogenated amorphous carbon

Pulsed vacuum arc deposition

Electrical resistivity

$\mathrm{sp}^{2}$ clusters

\begin{abstract}
A B S T R A C T
Electrically conductive amorphous carbon nitride (a-CN) coatings were deposited onto various substrates by pulsed vacuum-arc sputtering of a graphite cathode with simultaneous irradiation of the substrate with nitrogen ions. Deposition temperature was less than $60{ }^{\circ} \mathrm{C}$. We investigated the effect of vacuum annealing at a temperature of $600^{\circ} \mathrm{C}$ on the elemental composition, structure, electrical and optical properties of the obtained films. It was found that the elemental composition of the coating practically does not change over the thickness of the coating after annealing, while the remaining characteristics change significantly. Of particular interest is the correlation between the change in the nanostructure of the coating and the electrical properties of the coating. An explanation of the mechanisms for changing the properties of the coating after annealing is proposed.
\end{abstract}

\section{Introduction}

A wide range of physical, chemical, mechanical, tribological and biomedical properties of amorphous carbon coatings (a-C) is determined by the ratio of bonds of carbon atoms with $\mathrm{sp}^{1}, \mathrm{sp}^{2}$ and $\mathrm{sp}^{3}$ hybridization, as well as doping of films with various impurities [1,2]. Most publications are devoted to studies of the mechanical, tribological, and biomedical properties of a-C films, and only a small part of the work studies their electrical properties in detail. However, many practical applications require a-C coatings with high electrical conductivity, for example, for cantilevers used in current nanolithography [3]. Conductive carbon films are also considered a promising material for creating an alternative non-volatile memory with resistive switching [4].

Tetrahedral amorphous carbon films (ta-C), in which carbon atoms with $\mathrm{sp}^{3}$ hybridization predominate, are dielectrics with a resistivity of $10^{7}-10^{9} \Omega \mathrm{cm}$ at room temperature [5,6]. One of the most common ways to increase the electrical conductivity of ta-C is the introduction of nitrogen into their structure [5-11]. There are three main ways of introducing nitrogen into the carbon structure: inlet of molecular nitrogen $\mathrm{N}_{2}$ into the vacuum chamber during the deposition process [6,8-10]; deposition of a carbon coating with simultaneous irradiation with nitrogen ions [5,7,11]; - implantation of nitrogen ions into deposited carbon films [4]. The electrical properties of a-C coatings can only be controlled by introducing nitrogen at low deposition temperatures $\left(\leq 100{ }^{\circ} \mathrm{C}\right)$. Kleinsorge et al. [6] found that for tetrahedral carbon (ta-C) coatings the effect of nitrogen on the conductivity of the coating decreases with an increase in the deposition temperature, and above $200{ }^{\circ} \mathrm{C}$, the electrical conductivity does not depend on the nitrogen concentration. With increasing temperature, the amount of $\mathrm{sp}^{2}$ carbon fraction increases and the graphitization process is activated. Graphite-like films obtained by magnetron sputtering at a substrate temperature of $620^{\circ} \mathrm{C}$ have a resistivity of $\sim 0.03 \Omega \mathrm{cm}$, and the average $\mathrm{sp}^{2}$ crystallite size is $7.2 \mathrm{~nm}$ [12]. Given these temperature limitations, one of the most suitable methods for the deposition of a-CN coatings is pulsed vacuum-arc sputtering of graphite $[7,10,11]$. This method allows to obtain coatings of the required thickness by setting the desired number of pulses, to dose the thermal load on the substrate by changing the pulse repetition rate and does not require the application of an accelerating potential to the substrate. These advantages make it possible to deposit carbon coatings on dielectric and even polymer substrates without additional cooling. In practice, vacuum annealing has been successfully used to relieve internal compression stresses in ta-C coatings $[13,14]$. On the other hand, thermal annealing of a-CN coatings also leads to a decrease in their electrical resistivity [9], however, the structural changes that occur in this case, as well as the electrical conductivity mechanisms, are not fully understood.

\footnotetext{
* Corresponding author.

E-mail address: yuriyk@cinvestav.mx (Y. Kudriavtsev).
} 
This work is devoted to the study of the effect of vacuum annealing on the structural, electrical and optical properties of a conductive a-CN coating. At the same time, much attention is paid to studying the processes of atom clustering with the same type of coordination (with $\mathrm{sp}^{1}$, $\mathrm{sp}^{2}$, and $\mathrm{sp}^{3}$ hybrid states). The vacuum annealing temperature was chosen $600{ }^{\circ} \mathrm{C}$, since it is known that thermal annealing of ta-C coatings at this temperature occurs without changing the fraction of the $\mathrm{sp}^{3}$ phase $[13,14]$. Of particular interest is the comparison with the results obtained in Ref. [15], which describe local crystallization in a diamond-like carbon coating after annealing at $600{ }^{\circ} \mathrm{C}$.

\section{Experimental}

Coatings of a-CN were deposited from flows of carbon plasma and nitrogen ions incident on the substrate at an angle of $10^{\circ}-20^{\circ}$ to its surface. The carbon plasma flow was created by pulsed cathodic vacuum-arc sputtering of graphite with a purity of $99.99 \%$. The flow of nitrogen ions was created by an ion source with a cold cathode. At a discharge voltage of $0.5 \mathrm{kV}$, it forms a non-monoenergetic beam with an average ion energy of $100-250 \mathrm{eV}$. A detailed description of the coating production scheme can be found in Ref. [7]. The following materials were used as substrates: sitall, single crystal phosphorus doped silicon with (100) orientation, fused silica, $\mathrm{NaCl}$ salt. After loading the samples, the chamber was evacuated to a pressure of $3 \times 10^{-3} \mathrm{~Pa}$. Substrate temperature was less than $60^{\circ} \mathrm{C}$. The nitrogen pressure in the chamber was $0.2 \mathrm{~Pa}$. To identify thermally stimulated diffusion processes, a five-layer periodic coating was created on a silicon substrate: $\mathrm{C}-\mathrm{CN}-\mathrm{C}-\mathrm{CN}-\mathrm{C}$. All samples were divided into two parts, one of which was annealed.

Samples were annealed in an OTF-1200X-100 vacuum furnace at a temperature of $600^{\circ} \mathrm{C}$. The residual pressure in the furnace was $\sim 1 \mathrm{~Pa}$, the heating rate was approximately $15{ }^{\circ} \mathrm{C} / \mathrm{min}$, exposure at a given temperature of $10 \mathrm{~min}$, samples were taken from the furnace at a temperature of $<100^{\circ} \mathrm{C}$.

The elemental composition of a-CN coatings was determined by secondary ion mass spectrometry (SIMS). A TOF-SIMS-V time-of-flight mass spectrometer from ION-TOF GmbH (Germany) was used in the work. The depth profiling analysis was performed in a double-beam mode: the surface was sputtered $(300 \times 300 \mu \mathrm{m})$ with a beam of cesium ions with an energy of $2 \mathrm{keV}$; the central part of the sputter crater $(100 \times 100 \mu \mathrm{m})$ was analyzed using a pulsing source of bismuth ions $\mathrm{Bi}_{1}^{+}$ with an energy of $30 \mathrm{keV}$. Negative secondary ions were analyzed, separated by mass in a time-of-flight reflectron-type mass spectrometer. The experimental etching craters were measured with a Dektak XT, Bruker profilometer with an error of around 4\%. Quantitative analysis was performed using implanted standards.

The temperature dependences of the electrical resistivity of a-CN films in the range of 8-320 K were carried out using the van der Pauw method by using a Janis CCS-350S helium cryostat. The measurements were carried out on films with a thickness of $120 \mathrm{~nm}$ deposited on dielectric substrates of polished sitall with a size of $10 \times 10 \mathrm{~mm}$. The specific electric resistivity of the sitall is $\sim 10^{14} \Omega \mathrm{cm}$. The contacts to the sample were made using silver paste.

Transmission spectra were obtained from samples of a-CN films on fused silica in the range $200-1000 \mathrm{~nm}$ on an SF-2000 spectrophotometer. Raman spectra were recorded using a confocal Raman spectrometer HORIBA LabRAM HR Evolution (532 nm laser, 1800 gratings $/ \mathrm{mm}$, a $\times 50$ objective lens of $\mathrm{NA}=0.50$ ).

The structure of a-CN coatings was studied by Transmission Electron Microscopy (TEM) and Electron Energy Loss Spectroscopy (EELS) using a Tecnai G2 F20 S-TWIN transmission electron microscope with a Gatan 860 GIF 2001 sector-type post-column filter at an accelerating voltage of $200 \mathrm{kV}$. For TEM studies, a-CN coatings ( $\sim 30 \mathrm{~nm}$ thick) were deposited onto fresh cleaved $\mathrm{NaCl}$ followed by separation of the coating from the substrate in distilled water. TEM studies were carried out in bright-field and dark-field contrast of images with analysis of microdiffraction patterns. EELS measurements were performed with an energy resolution $1.8 \mathrm{eV}$ according to the FWHM of the zero-loss peak. K-edge spectra processing follows the standard procedure. The background was subtracted using an exponential function. Effects of plural scattering were removed by Fourier-ratio deconvolution with the relevant low-loss spectra.

The surface topography was analyzed by atomic force microscopy (AFM), using a NTEGRA AURA instrument (NT-MDT). The probe DCP10 have a nominal radius $\sim 10 \mathrm{~nm}$, a resonant frequency $\sim 200 \mathrm{kHz}$ and a force constant $\sim 10 \mathrm{~N} / \mathrm{m}$. The AFM image was processed according to ISO 25178-2: 2012.

\section{Results}

\subsection{SIMS}

According to the SIMS results, the ratio of the concentration of nitrogen to carbon (N/C) in the a-CN coating is $\sim 0.31-0.33$ and does not change after annealing in vacuum at a temperature of $600^{\circ} \mathrm{C}$. The presence of oxygen and hydrogen was also found, but their total concentration is less than 1 at.\%. In order to identify thermally stimulated diffusion processes, a study was made of a multilayer coating consisting of five alternating layers: $\mathrm{C}-\mathrm{CN}-\mathrm{C}-\mathrm{CN}-\mathrm{C}$. Nitrogen-free a-C layers were obtained by stopping the supply of nitrogen to the vacuum chamber. The a-C layer contains a large number of $\mathrm{sp}^{3}$ bonds, which determines its high electrical resistivity and density of about $2.79 \mathrm{~g} / \mathrm{cm}^{3}$ [7]. Fig. 1 shows the distribution profiles of nitrogen along the depth of the multilayer coating before and after annealing. It can be seen that the profiles are perfectly repeated, therefore, nitrogen is firmly connected to carbon and at this temperature there is no nitrogen diffusion inside the carbon matrix.

To examine the SIMS data, we made an elemental analysis of a 150 $\mathrm{nm}$ a:CN film, deposited under the same conditions on a silicon substrate by the EDX method. The annealing of these coatings was carried out for the same time and at the same temperature as the multilayer structures. The nitrogen concentration was determined to be 26.22 at. \% and 25.4 at. \% for the initial and annealed films, what is in a good coincidence with the SIMS data.

\subsection{Electrical resistance}

The electrical resistivity $\rho$ increases with decreasing temperature

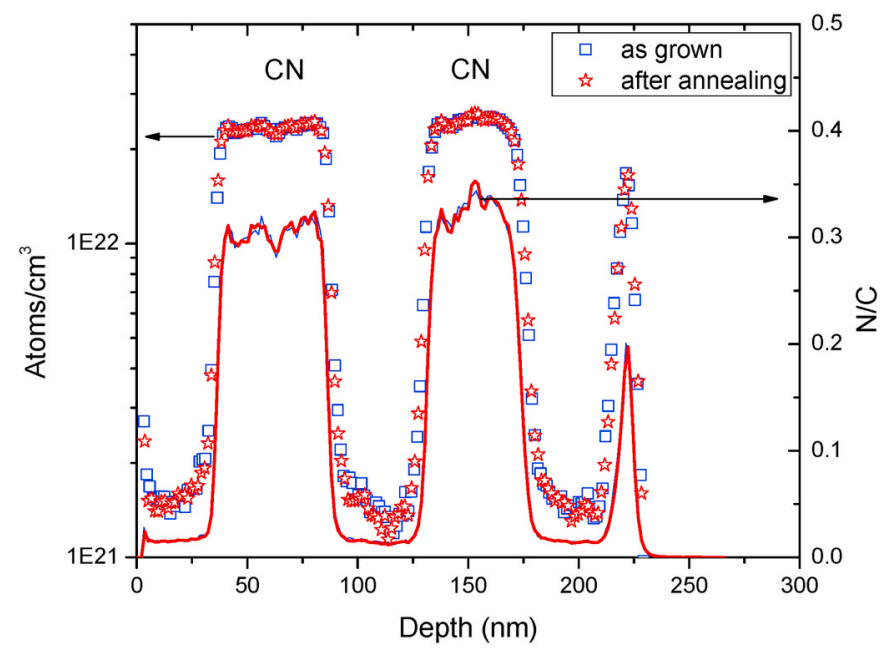

Fig. 1. Distribution profiles of nitrogen over the depth of the five-layer a-C/a$\mathrm{CN}$ coating before (rectangle) and after annealing at $600{ }^{\circ} \mathrm{C}$ (star). The right scale shows the ratio of nitrogen to carbon concentration as a percentage (lines). 


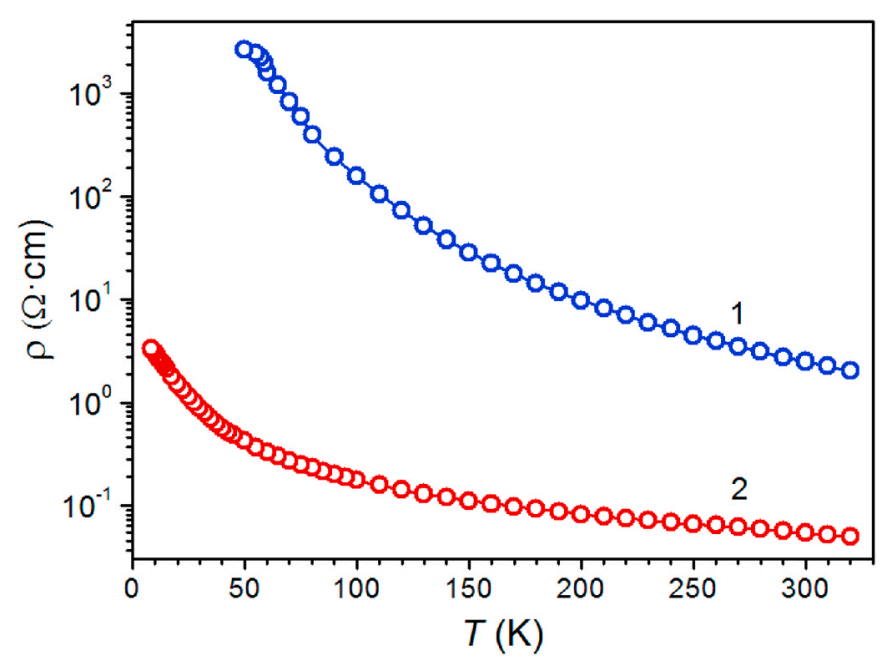

Fig. 2. Temperature dependence of the resistivity of a-CN covers before (1) and after (2) annealing.

(Fig. 2), which is typical for semiconductor structures. At room temperature $(300 \mathrm{~K})$, the specific resistivity of a-CN samples before and after annealing is 2.5 and $0.05 \Omega \mathrm{cm}$, respectively. That is, after annealing, $\rho$ decreased by 50 times. It is also seen that the change in $\rho$ with temperature in the annealed a-CN film is weaker than the initial film. A similar behavior was observed for ta-CN films in Ref. [9].

The resistivity of semiconductors in the thermal activation zone satisfies the equation:

$\rho(T)=\rho_{0} \exp \left(E_{A} / k T\right)$

where $\rho_{o}$ is the pre-exponential factor and $E_{A}$ is the activation energy.

Electrical conductivity in amorphous materials usually occurs by jumping an electron between localized states distributed in the mobility gap, either near the edge of the conduction band, near the Fermi level, or by conduction of electrons in extended states of the conduction band $[16,17]$.

To determine the mechanism of film conductivity, the temperature dependence of resistivity was investigated for compliance with the universal law [18]:

$\rho(T)=A T^{m} \exp \left(T_{0 j} / T\right)^{p}$

where $A$ is a constant and the exponents $m$ and $p$ are interrelated and depend on the mechanism of the hopping charge transfer; $T_{0}$ is a characteristic temperature.

Conductivity through the nearest neighbor hop (NNH) corresponds to the value of $p=1$, while the variable-range hopping (VRH) conductivity governed by the Mott and Shklovsky-Efros (SE) [19] mechanisms is characterized by $p=1 / 4, T_{0 j}=T_{0 M}$ or $p=1 / 2, T_{0 j}=T_{0 S E}$, respectively, where $T_{0 M}=\beta_{M} /\left[k g(\mu) a^{3}\right]$ and $T_{0 S E}=\beta_{S E} e^{2} /(k \kappa a)$. Here $\beta_{M}=21, \beta_{S E}=$ 2.8 and $g(\mu)$ is the density of the localized states (DOS) at the Fermi level, $k$ is the Boltzmann constant, $a$ is the localization radius of charge carriers, $e$ is the elementary charge and $\kappa$ is the dielectric constant. The parameter $\mathrm{m}$ can be calculated by the formula $m=(1-q) p$, where $q=$ 0 in the absence and $q=4$ in the presence of the contribution of a fluctuating short-range potential to microscopic disorder, respectively [18].

Typically, VRH conductivity occurs when the internal microscopic disorder is so high that tunneling between the nearest nodes becomes unlikely. Since at low $T$, electron hops occur only in a limited energy range near the Fermi level $(\mu)$, the type of charge transfer depends on the relationship between $\Delta \varepsilon(T)$ (the Mott optimal energy band decreasing with $T$ ), the width $W$ of the impurity band or the density of localized states (DLS), $g(\varepsilon)$, and the width $\Delta$ of the parabolic Coulomb gap. The latter occurs near $\mu$ as a result of the Coulomb interaction between charge carriers in disordered materials. For $\Delta<\Delta \varepsilon(T)<W$ and the weak dependence $g(\varepsilon)$, the Mott-VRH is established, while for $\Delta \varepsilon(T)<\Delta$, there is a VRH conductivity of type SE.

In the temperature interval where $T_{0 j}$ is constant, the local activation energy is: $E_{a}(T)=d \ln \rho(T) / d(k T)^{-1}[20]$.

Equation (2) can be presented in the form:

$\ln \left[E_{a} / k T+m\right]=\ln p+p \ln T_{0 j}+p \ln (1 / T)$

It can be seen the left-hand side of equation (3) is a linear function of $\ln (1 / T)$ for a given value of $m$ (for the case considered here $E_{a} / k T \gg m$ ). The value of parameter $p$ can be determined from the slope of the graph $\ln \left[E_{a} / k T+m\right]$ as a function of $\ln (1 / T)$ by the least square estimation (Fig. 3, insert). For both annealed and non-annealed a-CN films $p=0.25$ \pm 0.02 . It is typical for Mott mechanisms with a variable-range hopping. Note that the Hall effect was not observed in the a-CN coating, which may be due to the low number of delocalized states in this temperature range.

From Equation (2) it follows that the values $A$ and $T_{O M}$ can be found from linear segments of plots $\ln \left(\rho / T^{1 / 4}\right)$ versus $T^{-1 / 4}$ with previously determined $p$ and $m$ values (Fig. 3). Activation energy $E_{A}$ can be determined from the linear segment of the dependence $\ln \rho$ on $1 / \mathrm{T}$ in Eq. (1) at condition that coefficient $\rho_{o}$ weakly depend on $T$. The characteristic temperature $T_{O M}$ is determined by the density of localized states at the Fermi level $g(\mu)$ and the localization radius of charge carriers $a$ [20]. If we take $a=$ const [16], then the decrease of $T_{O M}$ after annealing is caused by an increase in $g(\mu)$. The values $\rho_{o}$ and $E_{A}$ are presented in Table 1.

\subsection{Optical transmission}

The initial a-CN coating $\sim 150 \mathrm{~nm}$ thick does not transmit ultraviolet radiation and weakly transmits in the visible region (Fig. 4). After annealing, the transmittance decreases significantly. The main parameter characterizing the optical properties of amorphous carbon films is the width of the optical gap $E_{g T}$ (Tauc gap) $[2,6,21,22]$, which is included in the well-known Tauc equation:

$\alpha E=B\left(E-E_{\mathrm{g} T}\right)^{2}$

where $E$ is the photon energy, $\alpha$ is the absorption coefficient, $B$ is a constant that is proportional to the density of states at the absorption edge of the amorphous semiconductor. The absorption coefficient $\alpha$ was calculated in accordance with the Lambert-Behr law (assuming no scattering). The spectral dependences $(\alpha E)^{1 / 2}$ of $E$ are presented in the insert of Fig. 4. Annealing of the a-CN film led to a decrease in $E_{g T}$ from $0.24 \mathrm{eV}$ to $0.01 \mathrm{eV}$, and to an increase in the parameter $B$ from 76320 to $88970(\mathrm{eV} \mathrm{cm})^{-1}$, which indicates an increase in the density of states at the absorption edge.

In our case, the Tauc formula satisfactorily describes the edge of optical absorption only in a certain spectral range. Therefore, it cannot be argued that the $E_{g T}$ value corresponds to the real gap width in the density of states of a-CN coatings. Most likely, it characterizes the minimum energy $\pi-\pi^{*}$ of the electronic transition [22]. For graphite, the width of the optical gap is zero, therefore, the tendency of $E_{g T}$ to zero should indicate the predominance of the $\mathrm{sp}^{2}$ phase in the coatings. At the same time, in Ref. [6], with an increase in the nitrogen concentration in ta-CN to $9 \%$, a decrease in the width of the optical gap to almost zero was observed at a constantly high content of the $\mathrm{sp}^{3}$ phase (about $80 \%$ ). Most likely, the closure of the optical gap is mainly due to the clustering of atoms with the $\mathrm{sp}^{2}$ bond, and not the transition of the $\mathrm{sp}^{3}$ phase to $\mathrm{sp}^{2}$.

In the $\pi$-bonded cluster model with planar aromatic clusters, the band gap depends on cluster size only [2,23]: $E_{g} \approx 2 \gamma / M^{1 / 2}$, where $\gamma$ is the nearest neighbor $\mathrm{V}(\mathrm{pp} \pi)$ interaction, and $M$ is the number of six-fold rings in the cluster. The parameter $\gamma=2.9 \mathrm{eV}$ for the $\pi$-type interaction between the nearest neighbors in the case of the $\mathrm{C}-\mathrm{C}$ bond [23]. 

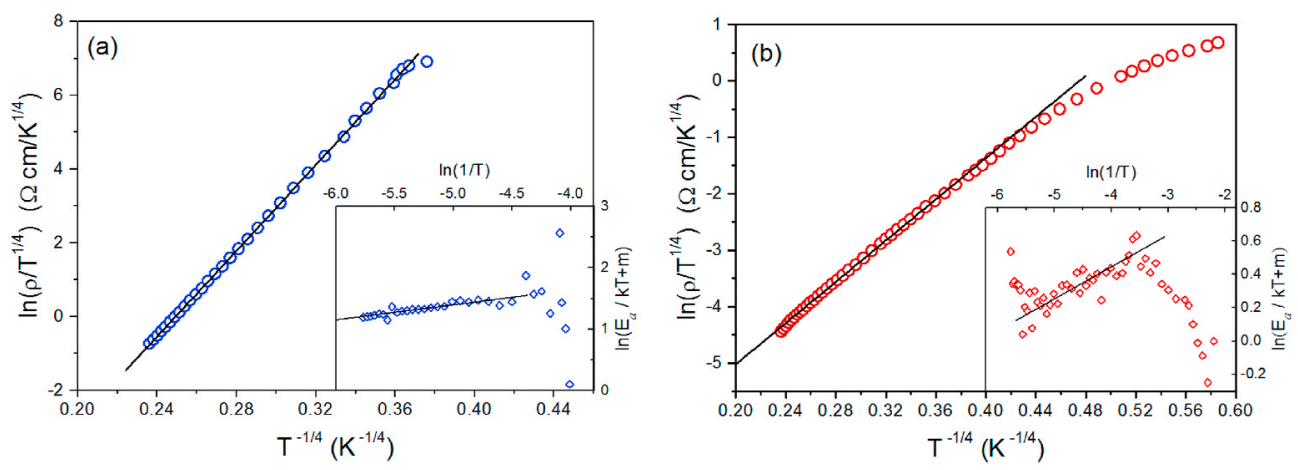

Fig. 3. Plots $\ln \left(\rho / T^{1 / 4}\right)$ versus $T^{-1 / 4}$ for a-CN cover before (a) and after (b) annealing. Insets: dependence of $\ln \left(E_{a} / k T+m\right)$ on $\ln (1 / T)$.

Table 1

Hopping conductivity parameters of a-CN films.

\begin{tabular}{lllll}
\hline a-CN & $\rho_{0}, \Omega \cdot \mathrm{cm}$ & $E_{A}, \mathrm{eV}$ & $A, \Omega \cdot \mathrm{cm} \cdot \mathrm{K}^{-1 / 4}$ & $T_{0}, \mathrm{~K}$ \\
\hline before annealing & 0.18 & 0.07 & $5.04 \times 10^{-7}$ & $1.2 \times 10^{7}$ \\
after annealing & 0.03 & 0.02 & $2.04 \times 10^{-4}$ & $0.9 \times 10^{5}$ \\
\hline
\end{tabular}

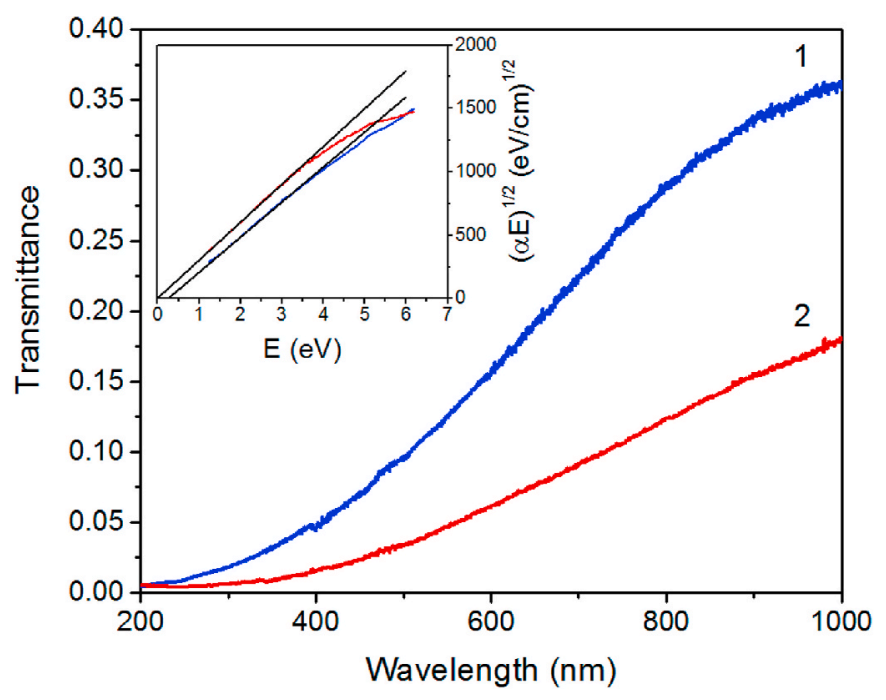

Fig. 4. Transparency of a-CN film before (1) and after (2) annealing.

However, for $E_{g T}<1 \mathrm{eV}$ this formula gives strongly overestimated cluster sizes, and for $E_{g T} \rightarrow 0$ the number of rings $M \rightarrow \infty$, which does not correspond to reality. In fact, the size of $\pi$-clusters is much smaller than follows from this model, $E_{g}$ is determined by distortions of $\pi$-states and cannot be described by a simple formula. In principle, the band gap itself depends only on the configuration of the $\mathrm{sp}^{2}$ sites, and this can vary independently of the $\mathrm{sp}^{2}$ concentration [24]. The authors of [25], for the case when $E_{g T} \leq 1 \mathrm{eV}$, propose using the parameter $B$ to estimate the concentration of sp ${ }^{2}$ clusters: $B \approx 5.2 \times 10^{-16} N_{0}$. For our samples, the initial concentration of $\mathrm{sp}^{2}$ clusters $N_{0}$ is $1.5 \times 10^{20} \mathrm{~cm}^{-3}$, and after annealing it increases to $1.7 \times 10^{20} \mathrm{~cm}^{-3}$.

\subsection{Raman spectroscopy}

It was shown in Ref. [26,27] that Raman spectra amorphous carbon nitride films can be explained in terms of an amorphous carbon-based model, without need of extra peaks due to vibration $\mathrm{CN}, \mathrm{NN}$, or $\mathrm{NH}$ modes. All amorphous carbons covers show common features in their Raman spectra in the $900-2000 \mathrm{~cm}^{-1}$ region: the G and D peaks, which lie at ca. 1560 and $1360 \mathrm{~cm}^{-1}$, respectively, for visible excitation, and the T peak at ca. $1060 \mathrm{~cm}^{-1}$, seen only in ultraviolet (UV) excitation. The $\mathrm{T}$ peak is due to the $\mathrm{C}-\mathrm{C} \mathrm{sp}^{3}$ vibrations. The $\mathrm{G}$ and $\mathrm{D}$ peaks are due to $\mathrm{sp}^{2}$ bonded carbon atoms. The $\mathrm{G}$ peak is due to the bond stretching of all pairs of $\mathrm{sp}^{2}$ atoms in both rings and chains. The $\mathrm{D}$ peak is due to the "breathing" modes of $\mathrm{sp}^{2}$ atoms in rings. Thus, no rings, no D peak.

Note that according to recent studies $[28,29]$, the peaks G and D are due to other mechanisms than described above. The $G$ peak which is nominally observed at $\sim 1580 \mathrm{~cm}^{-1}$ (if no stress shift is to be considered), does not correspond to $\mathrm{Csp}^{2}-\mathrm{Csp}^{2}$ stretching modes, but to local stationary phonon vibration modes of hexagonal cyclic $\mathrm{sp}^{2}$ rings. Moreover, the $\mathrm{G}$ band observed in amorphous materials is a superposition of the following structural peaks: $\mathrm{G}$ peak $\left(\sim 1580 \mathrm{~cm}^{-1}\right)$; the free $\mathrm{Csp}^{2}$ atom is bound to a $\mathrm{Csp}^{3}$ cluster $\left(\sim 1470 \mathrm{~cm}^{-1}\right)$; the free $\mathrm{Csp}^{3}$ atom is bound to the $\mathrm{Csp}^{2}$ cluster $\left(\sim 1560 \mathrm{~cm}^{-1}\right)$; C5 ring sp ${ }^{2}(\sim 1490$ $\left.\mathrm{cm}^{-1}\right)$; $\mathrm{C} 7$ ring sp ${ }^{2}\left(\sim 1540 \mathrm{~cm}^{-1}\right)$; $\mathrm{Csp}^{2}-\mathrm{Csp}^{2}$ clusters edge in a-C and DLC (sideband $\sim 1620 \mathrm{~cm}^{-1}$ ). The D erroneously so-called disorder peak which is observed at $\sim 1350 \mathrm{~cm}^{-1}$ (if no stress shift is to be considered) is not corresponding to a breathing mode in a hard carbon material because necessarily quenched by adjacent structures (only observed for free hexagonal cyclic $\mathrm{sp}^{2}$ structure). However, it corresponds to $\mathrm{Csp}^{2}-\mathrm{Csp}^{2}$ vibration mode of symmetric A-edge of $\mathrm{sp}^{2}$ clusters, meanwhile not for ZZ-edge. The T band corresponds to $\mathrm{Csp}^{3}$-Csp ${ }^{3}$ dangling bonds.

Characteristic G and D peaks of carbon are fitted using the Breit-Wigner-Fano (BWF) and Lorentzian fit, respectively (Fig. 5). The BWF line for the $G$ peak has an asymmetric line shape, which arises from the coupling of a discrete mode to a continuum. A Lorentzian line shape is used for the D peak as it is from the same family as the BWF line. The BWF line shape is given by Refs. [30]:

$I(\omega)=\frac{I_{0}\left[1+2\left(\omega-\omega_{0}\right) / Q \Gamma\right]^{2}}{1+\left[2\left(\omega-\omega_{0}\right) / \Gamma\right]^{2}}$

where $I_{0}$ is the peak intensity, $\omega_{0}$ is the peak position, $\Gamma$ is assumed as the full width at half maximum (FWHM) and $Q^{-1}$ is the BWF coupling coefficient. The maximum of the BWF line is not at $\omega_{0}$ but lies at lower frequencies: $\omega_{\max }=\omega_{0}+\Gamma / 2 Q$, as $Q$ is negative. We define the $\mathrm{G}$ position as $\omega_{\max }$ rather than $\omega_{0}$. The decomposition of the experimental Raman spectra into $\mathrm{G}$ and $\mathrm{D}$ peaks was performed using the Origin software package. The decomposition window is $900-2000 \mathrm{~cm}^{-1}$ for an unannealed sample, and $950-1950 \mathrm{~cm}^{-1}$ for an annealed sample.

For the initial a-CN coating: $P_{\text {os }}(G)=1554 \mathrm{~cm}^{-1}$, FWHM $(G)=213$ $\mathrm{cm}^{-1}, \mathrm{P}_{\text {os }}(\mathrm{D})=1366 \mathrm{~cm}^{-1}$, FWHM (D) $=327 \mathrm{~cm}^{-1}, \mathrm{I}(\mathrm{D}) / \mathrm{I}(\mathrm{G})=0.70$ (Fig. 5a). After annealing: $P_{o s}(G)=1564 \mathrm{~cm}^{-1}$, FWHM $(G)=173 \mathrm{~cm}^{-1}$, $\mathrm{P}_{\mathrm{os}}(\mathrm{D})=1343 \mathrm{~cm}^{-1}$, FWHM (D) $=298 \mathrm{~cm}^{-1}$, I (D) $/ \mathrm{I}(\mathrm{G})=1.06$ (Fig. 5b). Annealing leads to a shift in the position of the peak $G$ toward higher wave numbers, an increase in the intensity of the peak $\mathrm{D}$ and the ratio I (D)/I(G), while the FWHM of both peaks decreases. These changes in the Raman spectrum are caused by the clustering of atoms having $\mathrm{sp}^{2}$ hybridization [30]. Therefore, annealing favors the rearrangement of the 

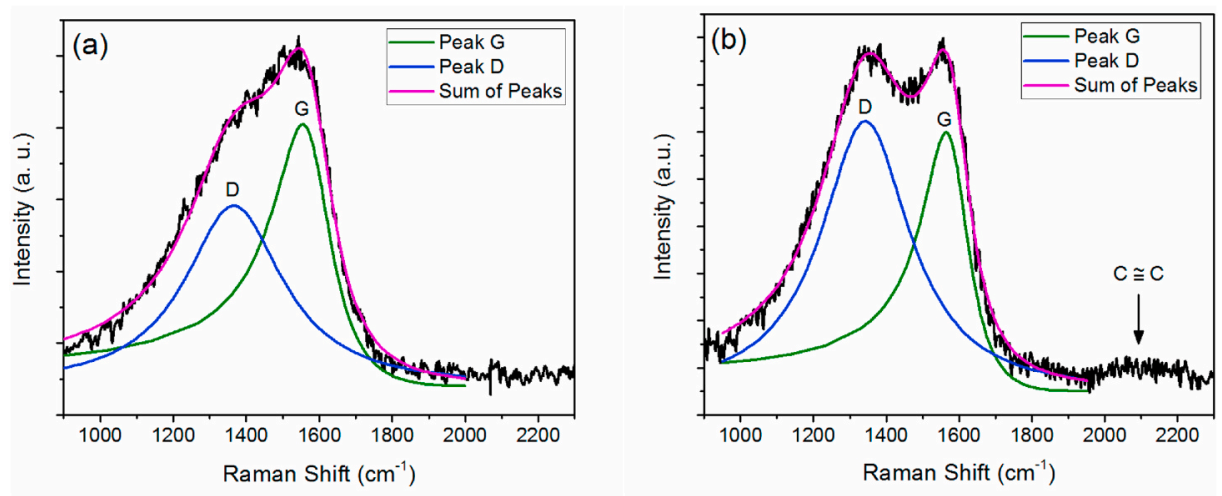

Fig. 5. The fit of the Raman spectra of the a-CN before (a) and after annealing (b) consists of the BWF line for peak G and Lorentz for peak D.

amorphous matrix, which leads to an increase in the number of closed carbon rings in $\mathrm{sp}^{2}$ clusters and (or) the number of clusters containing rings. The average cluster size can be estimated by the dependence of I (D)/I(G) on the cluster diameter [30]. According to the estimation results, the average size of the $\mathrm{sp}^{2}$ cluster in the initial coating is $1.1 \mathrm{~nm}$, and after annealing it increases to $1.4 \mathrm{~nm}$. According to the three-stage model of the transition (amorphization trajectory) from ideal graphite to ta-C $[26,27,30]$, the structure of our a-CN coating corresponds to stage 2 (reverse transition a-C $\rightarrow$ nanocrystalline graphite (nc-G)).

A weak band appears in the spectrum of annealed a-CN in the region of $1950-2250 \mathrm{~cm}^{-1}$, which is characteristic of carbon chains of the polyyne $(-\mathrm{C} \equiv \mathrm{C}-)$ and cumulene $(=\mathrm{C}=\mathrm{C}=$ ) type $[27,31]$. Although it is known that carbon chains with $\mathrm{sp}^{1}$ hybridization have high chemical activity and are oxidized in air [32]. On the other hand, in Ref. [33] stable hexagonal carbyne crystals $(-\mathrm{C} \equiv \mathrm{C}-$ ) were obtained in the form of a white powder, which has two sharp peaks in the Raman spectrum at $1050 \mathrm{~cm}^{-1}$ (due to single $\mathrm{C}-\mathrm{C}$ bonds) and at $2175 \mathrm{~cm}^{-1}$ (due to triple $\mathrm{C} \equiv \mathrm{C}$ bonds). As for the nitrile type bonds $(-\mathrm{C} \equiv \mathrm{N})$, the characteristic band for them is not observed in the region of $2220-2270 \mathrm{~cm}^{-1}$.

\subsection{TEM and EELS}

In Fig. 6 shows brightfield TEM images of a-CN before and after annealing. Before annealing, the a-CN film has a fairly uniform appearance, which is characteristic of amorphous carbon structures [34]. After annealing, we observe clear structural changes associated with the formation of more ordered clusters.

Electron microscopic studies of a-CN coatings in electron diffraction mode showed that vacuum-annealed coatings contain regions of ordered nanoclusters. For an a-CN sample without annealing (initial), microdiffraction is wide diffuse rings (Fig. 6a, insert). The microdiffraction of the annealed sample shows a quasi-circular character of the distribution of reflections, which is characteristic of materials with numerous dispersed nanosized inclusions (Fig. 6b, insert).

Dark-field TEM images are used to visualize ordered structures (clusters and nanocrystals) in an amorphous matrix [35] The nanoscale clusters were observed by using conventional TEM dark field technique, which has been being applied for measurements of dispersed particle size [36]. Note that the measurements of the cluster sizes are of an estimate nature. The dark-field images of annealed a-CN coating obtained in the region of the ring distribution of reflections (indicated by the arrow in Fig. 6b) show a contrast characteristic of ordered nanoscale clusters (Fig. 7a.). For quantitative assessment of cluster sizes, the image was binarized with subsequent statistical processing. Most clusters range in size from 3.5 to $10 \AA$ (Fig. 7b). Clusters are not observed on TEM images of the initial sample (without annealing) obtained in dark-field contrast.

It is important to note that when studying the coating using a transmission electron microscope, no ordered micro or nanocrystals were detected, as was previously observed in Ref. [15].

Fig. 8 shows the EELS spectra of the K-edge elements of the matrix of a-CN coatings before and after annealing. At the K-edge of carbon, we observe two peaks. A wide structureless peak above $292 \mathrm{eV}$ corresponds to $1 \mathrm{~s} \rightarrow \sigma^{*}$ electron transitions in $\mathrm{C}$ atoms with any type of hybridization. The $\pi^{*}$ peak with a maximum of about $285.5 \mathrm{eV}$ corresponds to $1 \mathrm{~s} \rightarrow \pi^{*}$ transitions, and its presence is usually associated with the presence in
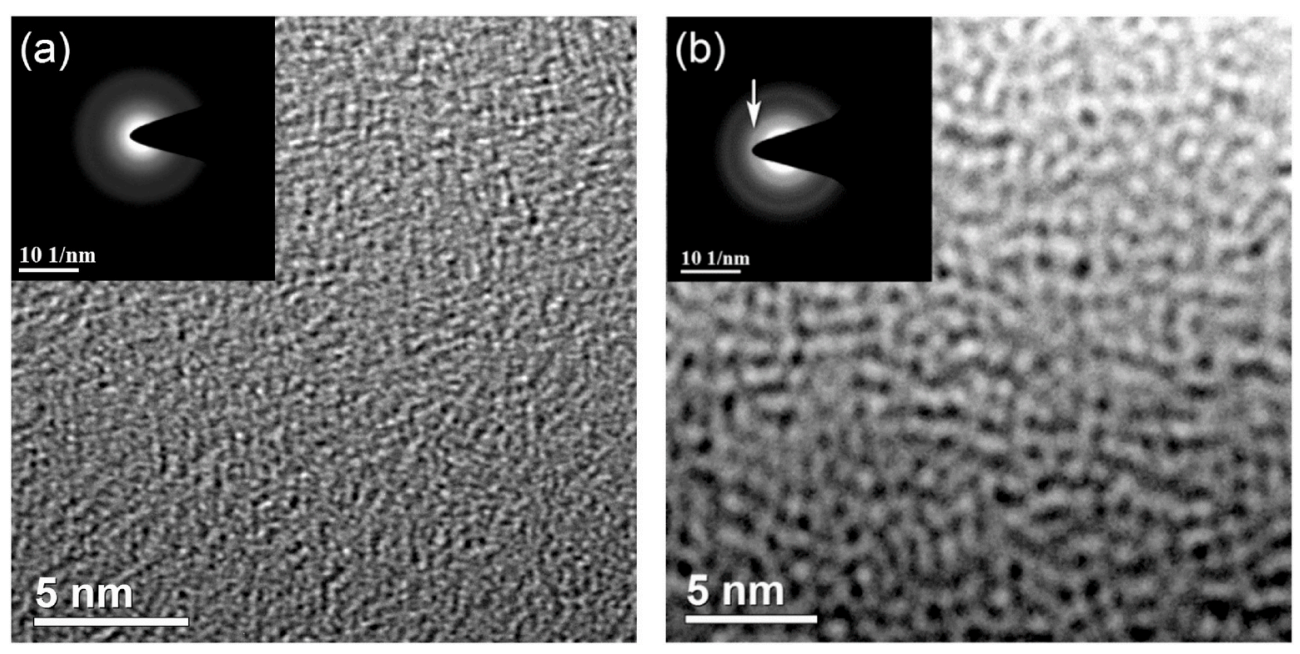

Fig. 6. Plan view TEM images of a-CN before (a) and after (b) annealing. On the diffractogram insertion. The diffraction rings for the annealed a-CN coating correspond to the interplanar spacings of $1.96 \pm 0.21 \AA$ and $1.04 \pm 0.04 \AA$. 

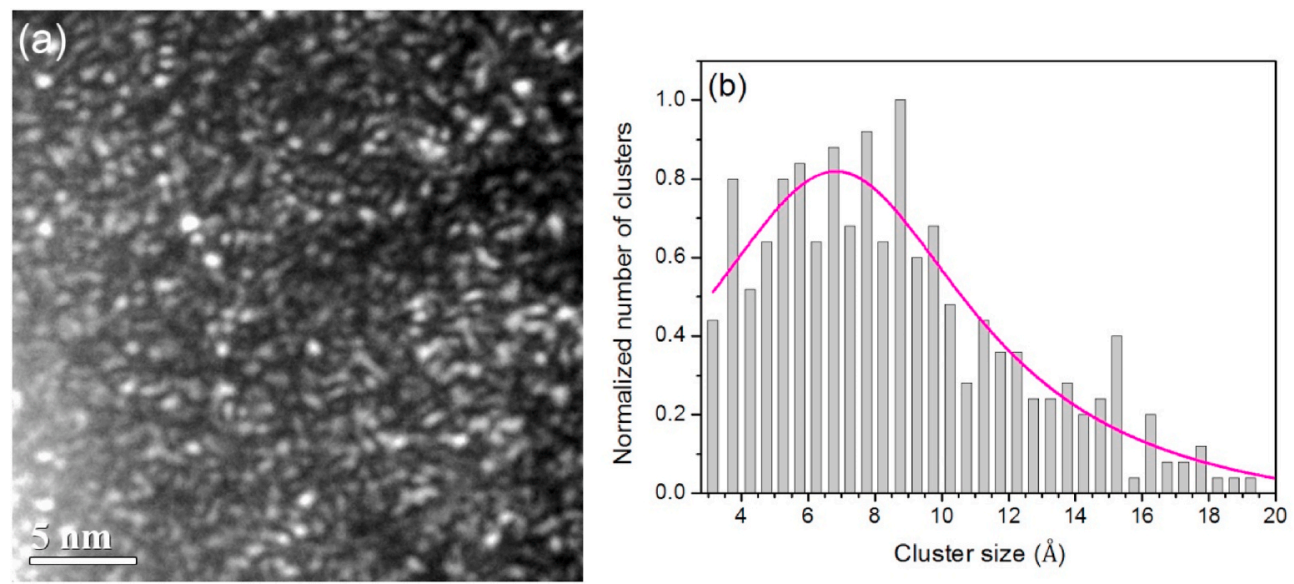

Fig. 7. Dark-field TEM image of annealed a-CN coating (a), and cluster size distribution (b). The shooting area is indicated by an arrow on the diffractogram (Fig. 6b, insert).
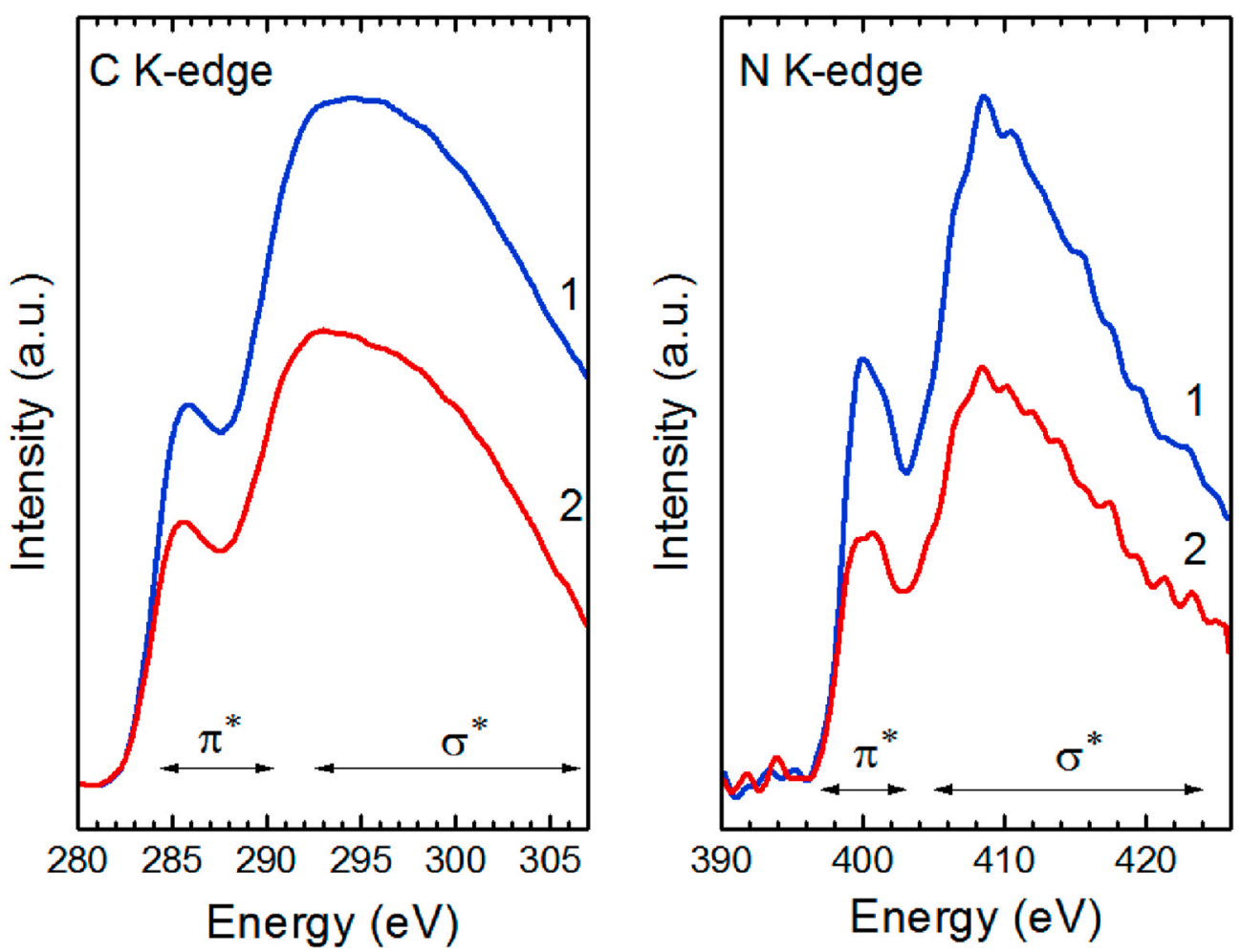

Fig. 8. Carbon and nitrogen K-edge spectra of a-CN before (1) and after (2) annealing.

the matrix of atoms with.

$\mathrm{sp}^{2}$ hybridization of electronic orbitals, ignoring the contribution from atoms with $\mathrm{sp}^{1}$ hybridization. In the spectrum of the $\mathrm{K}$ edge of nitrogen, we also observe two peaks $\pi^{*}$ and $\sigma^{*}$. The peak position $\pi^{*}$ $(\sim 400 \mathrm{eV})$ is below the ionization energy characteristic of nitrogen (401 $\mathrm{eV}$ ). A region with energies above $406 \mathrm{eV}$ corresponds to $1 \mathrm{~s} \rightarrow \sigma^{*}$ transitions of electrons in $\mathrm{N}$ atoms. In the spectrum of $\mathrm{N}$, the peak shape $\pi^{*}$ indicates that it consists of at least two component peaks, the intensity ratio of which changes after annealing. Due to the insufficient energy resolution of the EELS method, the obtained spectra lack a detailed fine structure of the density of unoccupied states, which does not allow us to unambiguously determine the $\mathrm{CN}$ bond form.

However, the near edge X-ray absorption fine structure (NEXAFS) method with a resolution of $\sim 0.1 \mathrm{eV}$ showed that the $\pi^{*}$ peak for amorphous a-CN structures [37-39] and even for nitrogen-doped carbon nanotubes [40] is indeed a superposition of various types of $\mathrm{CN}$ bonds. The most likely forms of nitrogen bonding to carbon are: $\mathrm{sp}^{3} \mathrm{~N}$ bonded to $\mathrm{sp}^{3} \mathrm{C}$ in "open" structures; $\mathrm{sp}^{3} \mathrm{~N}$ bonded to $\mathrm{sp}^{3} \mathrm{C}$ in a closed structure in which $\mathrm{C}-\mathrm{N}-\mathrm{C}$ bonds are stressed; $\mathrm{sp}^{1} \mathrm{~N}$ in nitrile-like structures; $\mathrm{sp}^{2} \mathrm{~N}$ in pyridine-like structures; $\mathrm{sp}^{2} \mathrm{~N}$ substituting $\mathrm{sp}^{2} \mathrm{C}$ in graphite-like structures [41].

The two-windows method with the optimized settings of energy-loss integration windows for the $\pi^{*}$ and $\sigma^{*}$ states was used to estimate the fraction of $\mathrm{sp}^{2}$-hybridized carbon atoms [42]. For the peak $\pi^{*}$, the width of the integration window is $0.6 \mathrm{eV}$ relative to the center, for the peak $\sigma^{*}$, the integration window is between 292 and $307 \mathrm{eV}$. Highly ordered pyrolytic graphite (HOPG) was adopted as the reference material. The content of carbon atoms with $\mathrm{sp}^{2}$ hybridization in the initial a-CN coating is $63 \%$, in the annealed $69 \%$. Assuming the absence of carbon atoms with $\mathrm{sp}^{1}$ hybridization, the proportion of the $\mathrm{sp}^{3}$ phase is $37 \%$ and 
$31 \%$ before and after annealing, respectively.

\subsection{Atomic force microscopy}

The surface topography was investigated on samples of a-CN coatings deposited on sitall substrates. Fig. 9 shows the AFM image of the surface of the a-CN coating before annealing, AFM image of the surface of the a-CN coating after annealing has a similar form. Table 2 shows the results of the analysis of its surface before and after annealing. AFM shows that annealing of the coating does not change the surface roughness. The average root-mean-square (RMS) roughness of the 120 $\mathrm{nm}$ thick coatings is 6 times higher than the initial roughness of the sitall substrate. The values of $S 3 A, S_{d r}, S_{d s}$ and $d$ do not change after annealing at a temperature of $600{ }^{\circ} \mathrm{C}$ in vacuum.

\section{Discussion}

SIMS results together with EELS results let us conclude that in our a$\mathrm{CN}$ coating, nitrogen is strongly bonded to carbon. We have obtained a multilayer $(-\mathrm{C}-\mathrm{CN}-)$ coating, in which there is no nitrogen diffusion during thermal annealing up to $600^{\circ} \mathrm{C}$, this opens up the possibility of creating two-layer or multi-layer coatings based on solid, wear-resistant, dielectric ta-C and electrically conductive a-CN. Such coatings will be in demand in various fields of science and technology. For example, to create an effective thermoelectric converter based on a three-layer carbon structure dielectric-conductor-dielectric [43]. Conductive periodic ta-C/a-CN eight-layer films have potential application as protective films for contact pins used in the electrical inspection process for integrated circuit chips [44]. In this case, subsequent annealing in vacuum should significantly increase the electrical conductivity of a-CN layers and relieve internal compression stresses in ta-C layers without deterioration of operational properties. It is worth noting that in Ref. [45] pure (without $\mathrm{N}$ ) carbon coatings were obtained, which are layers of low density ( $\mathrm{sp}^{2}$ rich) and high density $\left(\mathrm{sp}^{3}\right.$ rich) with a clear interface between them. However, according to the TEM and EELS data after vacuum annealing at $600{ }^{\circ} \mathrm{C}$ for $10 \mathrm{~min}$, the boundary between the layers disappeared, the low-density regions became more oriented, and the high-density regions were transformed into low-density material [45].

The presence of a $\pi^{*}$ peak in the EELS spectra, a peak D in the Raman spectra, low electrical resistivity, and a narrow width of the optical gap

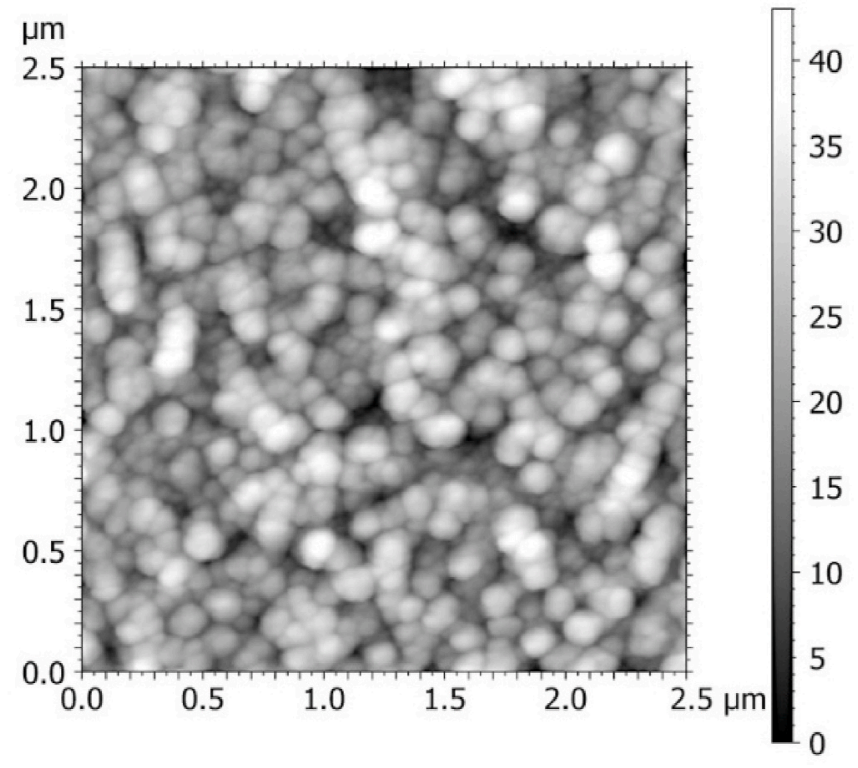

Fig. 9. AFM image the surface of a-CN coating before annealing. indicate the predominance of the $\mathrm{sp}^{2}$ phase in a-CN. However, the quantitative estimation of the $\mathrm{sp}^{2}$ phase (63\%), performed on the K-edge carbon spectra of EELS, can be overestimated. After annealing of a-CN, a weak band appeared in the Raman spectrum, which is characteristic for the carbon chains of cumulene $(=\mathrm{C}=\mathrm{C}=$ ) and (or) polyyne type $\left(-\mathrm{C} \equiv \mathrm{C}-\right.$ ). If a- $\mathrm{CN}$ contains a sp ${ }^{1}$ bond, as in the indicated structures, or as in nitrile groups $(-\mathrm{C} \equiv \mathrm{N})$, then the $\pi^{*}$ peak corresponds to the sum of $\mathrm{sp}^{2}$ sites plus twice the $\mathrm{sp}^{1}$ sites (which have two $\pi$ states). In our Raman spectra, the band characteristic of nitrile groups is not observed, but this does not mean that nitrile groups are completely absent. The fact is that they are mainly observed upon ultraviolet excitation, since the band gap of the $\pi-\pi^{*}$ transition is $5-6 \mathrm{eV}$ [26]. Depending on the technological parameters of deposition and nitrogen concentration, the content of nitrile groups can reach 18\% [46]. We, like other researchers [37-39], believe that the intense $\pi^{*}$ peak at the K-edge of nitrogen in the EELS spectra is mainly due to $\mathrm{sp}^{2}$-hybridized nitrogen atoms. Let us now look at the appearance in the Raman spectrum of the annealed a-CN coating of a band characteristic of carbon chains. In Ref. [31], Raman spectra of molecules consisting of single carbon chains of cumulene and polyyne type, as well as double chains with a bend between linear fragments, were simulated. Calculations showed that only single cumulenes consisting of 3 and 5 carbon atoms in a molecule have an intensity of vibrational modes close to zero above $2000 \mathrm{~cm}^{-1}$. Therefore, structures of the type $(=\mathrm{C}=\mathrm{C}=$ ) can theoretically be present in the initial a-CN matrix. As a result of thermal exposure, an increase in the length of cumulene-like chains and (or) the formation of polyyne-like chains ( $-\mathrm{C} \equiv \mathrm{C}-$ ) can occur, which leads to the appearance of the corresponding band in the Raman spectrum. In this regard, the suggested increase in the fraction of carbon atoms with $\mathrm{sp}^{2}$ hybridization from $63 \%$ to $69 \%$ as a result of annealing can in fact be partially related to the increase in the fraction of $\mathrm{sp}^{1}$ hybridized atoms.

According to the cluster model [2,23], carbon atoms with $\mathrm{sp}^{2}$ hybridization tend to unite into flat $\pi$ bound clusters embedded in the $\mathrm{sp}^{3}$ matrix. The formation of these clusters is due to the fact that $\mathrm{sp}^{2}$ orbitals located close to the Fermi level $\left(E_{F}\right)$ acquire stability as a result of an increase in the interaction energy between $\pi$ orbitals. In this case, any cluster seeks to have an even number of $\pi$ orbitals; otherwise, a half-filled state will be on $E_{F}$, which will lower the binding energy. An estimate based on optical spectroscopy showed that the number of $\mathrm{sp}^{2}$ clusters in the a-CN coating is $1.5 \times 10^{20} \mathrm{~cm}^{-3}$, and after annealing it increases to $1.7 \times 10^{20} \mathrm{~cm}^{-3}$. Note that a significant number of clusters already exist in the initial coating, and annealing leads to internal ordering and growth of existing clusters, as well as the formation of new ones. Using high-resolution TEM, we were able to directly measure the size of these clusters (Fig. 6). For annealed a-CN, the cluster size lies in the range 3.5-19.5 $\AA$. Note that the cluster size determined by the ratio I (D)/I(G) of the Raman spectrum (14 $\AA$ ) falls into the indicated range. Suppose that the $\mathrm{sp}^{2}$ cluster is a flat circle with a diameter $D$. Now we can estimate the number of carbon rings in it as the number of regular hexagons inscribed in a circle with a diameter $D$. In this approximation, the number of hexagonal rings with side $b=1.41 \AA$ contained in a flat cluster is determined by the formula $M=\pi D^{2} / 6 \sqrt{3} b^{2}$. According to our results, clusters contain from 2 to 58 rings. According to the Hückel rule, $N_{\pi}=2(2 M+1)$, the cluster contains from 10 to $234 \pi$ electrons, depending on its size. Let us estimate the concentration of atoms in the composition of all clusters $N=6 M N_{0}$. Suppose that all clusters have a size of $D=10 \AA$, then $M=15, N_{0}=1.7 \times 10^{20} \mathrm{~cm}^{-3}$, and $N=1.5 \times 10^{22}$ atoms $\cdot \mathrm{cm}^{-3}$. It can be seen that quite real values are obtained that are comparable with the density of the material, at least in order of magnitude.

Our a-CN coatings are Mott semiconductors up to room temperature, which is consistent with previous studies of the conductivity of pure and doped a-C $[9,16]$. Moreover, they are characterized by a very narrow optical gap. We use the cluster model $[2,23]$, considering that $\mathrm{sp}^{2}$ bonds act as localized conduction states [16]. In the electronic structure of 
Table 2

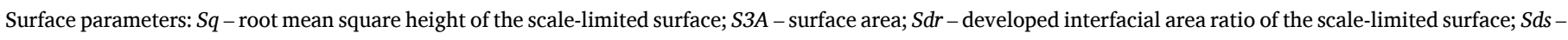
density of summits of the surface; $d$ - average lateral diameter of summits of the surface.

\begin{tabular}{|c|c|c|c|c|c|c|}
\hline Sample & & $S q(\mathrm{~nm})$ & $S 3 A \mu \mathrm{m}^{2}$ & $S_{d r}(\%)$ & $\mathrm{S}_{\mathrm{ds}}\left(1 / \mu \mathrm{m}^{2}\right)$ & $d(\mathrm{~nm})$ \\
\hline Sitall substrate & & 1.2 & 6.26 & - & - & - \\
\hline \multirow[t]{2}{*}{$\mathrm{a}-\mathrm{CN}$} & before annealing & 6.9 & 6.42 & 2.9 & 92 & 83.1 \\
\hline & after annealing & 7.2 & 6.46 & 3.4 & 99 & 77.2 \\
\hline
\end{tabular}

amorphous carbon, the localized states $\sigma$ and $\sigma^{*}$ form deep levels in the valence and conduction bands, respectively. Inside the $\sigma-\sigma^{*}$ - gap there are states $\pi$ and $\pi^{*}$, which form the edge of the absorption band and control the width of the optical gap. The filled valence band is formed by $\pi$ states, and the empty conduction band corresponds to $\pi^{*}$ states near the Fermi level. Therefore, together with an increase in $\mathrm{sp}^{2}$ bonds, the number of localized states near the Fermi level also increases, which we observe during thermal annealing. The closure of the optical gap $E_{g T} \rightarrow$ 0 and an increase in the parameter $B$ upon annealing are caused by an increase in the size and number of $\mathrm{sp}^{2}$ clusters, and hence an increase in the density of localized states $g(\mu)$ near the Fermi level. Similarly, a decrease after annealing of the parameters $T_{0}$ (Table 1 ) is a consequence of an increase in $g(\mu)$, which leads to a decrease in hopping resistance. A decrease in the activation energy $E_{A}$ indicates a partial ordering of the structure. The $\mathrm{sp}^{3}$ hybridization phase has a wide band gap, thereby creating a tunnel barrier between $\mathrm{sp}^{2}$ clusters. The presence in the coating matrix of $\mathrm{sp}^{1}$ chains, for example of the type $(=\mathrm{C}=\mathrm{C}=)$, can create a kind of network of conducting percolation channels between $\mathrm{sp}^{2}$ clusters, thereby reducing the total resistivity.

In conclusion, we note that the electrical resistivity of our a-CN coatings ( $\rho=2.5 \Omega \mathrm{cm}$ at $T=300 \mathrm{~K}$ ) is 4 times lower than that of analogs $[5,11]$, also obtained by cathodic vacuum-arc sputtering of graphite with simultaneous irradiation with nitrogen ions of the growing film. Subsequent annealing in vacuum at $600{ }^{\circ} \mathrm{C}$ makes it possible to reduce the electrical resistivity to $0.05 \Omega \mathrm{cm}$, which is commensurate with the resistance of nanocrystalline graphite films to $\sim 0.03 \Omega \mathrm{cm}$ [12]. At the same time, "fullerene-like" carbon nitride films $\mathrm{CN}_{0.2}$ consisting of curved, frequently intersecting, and highly in-plane oriented basal lattice planes, deposited by unbalanced reactive magnetron sputtering at $350{ }^{\circ} \mathrm{C}$, have resistivity an order of magnitude lower than $0.004 \Omega \mathrm{cm}$ [47].

\section{Conclusions}

The a-CN ultronanocluster electrically conductive coatings ( $\rho=2.5$ $\Omega \mathrm{cm}$ at $T=300 \mathrm{~K}$ ) were obtained by the method of deposition of carbon from a pulsed vacuum arc plasma with simultaneous irradiation with nitrogen ions at a substrate temperature $<60^{\circ} \mathrm{C}$. Clusters are formed by $\mathrm{sp}^{2}$-bonded carbon and nitrogen atoms, and nitrogen is strongly bonded to carbon. Based on the results of studies of structural, electrical, and optical properties, the size and concentration of clusters are determined. It is shown that it is the electrical properties that are most sensitive to any structural changes. Additional annealing in vacuum at $600{ }^{\circ} \mathrm{C}$ allows a 50-fold decrease in the electrical resistivity of the a-CN coating. In this case, the fraction of $\mathrm{sp}^{3}$-bonded carbon atoms in the coating matrix is at least $31-37 \%$. The electrical and optical properties are mainly determined by the size, quantity, and location of $\mathrm{sp}^{2}$ clusters.

The increase in conductivity after annealing is due to the following processes: ordering inside $\mathrm{sp}^{2}$ clusters; increase in cluster size; increase in their number; the formation between the clusters of a network of conducting percolation channels. In the Raman spectra of annealed a$\mathrm{CN}$, exhibit a weak band is characteristic of $\mathrm{sp}^{1}$ structures.

It was found that vacuum annealing at $600^{\circ} \mathrm{C}$ does not lead to changes in the surface relief of the a-CN coating.

The results can be used to create conductive coatings, as well as multilayer systems based on them for various fields of science and technology, this necessitates further studies aimed at studying the effect of vacuum annealing on the mechanical and tribological properties of electrically conductive single-layer a-CN, and periodic ta-C/a-CN multilayer systems. A separate difficult scientific task is the quantitative estimation of the fraction of atoms with $\mathrm{sp}^{1}$ hybridization of electronic orbitals.

\section{Declaration of competing interest}

The authors declare that they have no known competing financial interests or personal relationships that could have appeared to influence the work reported in this paper.

\section{References}

[1] J. Vetter, 60 years of DLC coatings: historical highlights and technical review of cathodic arc processes to synthesize various DLC types, and their evolution for industrial applications, Surf. Coat. Technol. 257 (2014) 213-240, https://doi.org/ 10.1016/j.surfcoat.2014. 08.017.

[2] J. Robertson, Diamond-like amorphous carbon, Mater. Sci. Eng., R 37 (2002) 129-281, https://doi.org/10.1016/S0927-796X(02)00005-0.

[3] A.Ya Kolpakov, A.I. Poplavsky, O.V. Safronova RU, Patent 136563 U1, 2013.

[4] V.K. Nagareddy, A.K. Ott, C. Dou, T. Tsvetkova, M. Sandulov, M.F. Craciun, A. C. Ferrari, C.D. Wright, The effect of nitrogen implantation on resistive switching of tetrahedral amorphous carbon films, Diamond Relat. Mater. 87 (2018) 90-98, https://doi.org/10.1016/j.diamond. 2018.05.005.

[5] L.K. Cheah, X. Shi, J.R. Shi, E.J. Liu, S.R.P. Silva, Properties of nitrogen doped tetrahedral amorphous carbon films prepared by filtered cathodic vacuum arc technique, J. Non-Cryst. Solids 242 (1998) 40-48, https://doi.org/10.1016/S00223093(98)00787-X.

[6] B. Kleinsorge, A.C. Ferrari, J. Robertson, W.I. Milne, Influence of nitrogen and temperature on the deposition of tetrahedrally bonded amorphous carbon, J. Appl. Phys. 88 (2000) 1149-1157, https://doi.org/10.1063/1.373790.

[7] A.I. Poplavsky, A.Ya Kolpakov, Yu Kudriavtsev, R. Asomoza, I.Yu Goncharov, M. E. Galkina, S.S. Manokhin, V.A. Kharchenko, Effect of nitrogen ion irradiation parameters on properties of nitrogen-containing carbon coatings prepared by pulsed vacuum arc deposition method, Vacuum 152 (2018) 193-199, https://doi. org/10.1016/j.vacuum.2018.03.028.

[8] Sekhar C. Ray, W. Mbiombi, P. Papakonstantinou, Electrical and electronic properties of nitrogen doped amorphous carbon $\left(\mathrm{a}-\mathrm{CN}_{\mathrm{x}}\right)$ thin films, Curr. Appl. Phys. 14 (2014) 1845-1848, https://doi.org/10.1016/j.cap.2014.10.016.

[9] Z.Y. Chen, Y.H. Yu, J.P. Zhao, S.Q. Yang, T.S. Shi, X.H. Liu, E.Z. Luo, J.B. Xu, I. $\mathrm{H}$. Wilson, Electrical properties of nitrogen incorporated tetrahedral amorphous carbon films, Thin Solid Films 339 (1999) 74-77, https://doi.org/10.1016/S00406090(98)01066-9.

[10] A. Ya Kolpakov, I.V. Sudzhanskaya, M.E. Galkina, I. Yu Goncharov, A.I. Poplavskii, S.S. Manokhin, Nanometer-sized carbon coatings on a silicon wafer: the effect that nitrogen doping level has on specific conductivity and morphology, Nanotechnologies in Russia 6 (2011) 185-188, https://doi.org/10.1134/ S199507801102011X.

[11] I.A. Zavidovskii, O.A. Streletskii, O.Y. Nishchak, A.A. Khaidarov, The effect of the ion assistance energy on the electrical resistivity of carbon films prepared by pulsed plasma deposition in a nitrogen atmosphere, Phys. Solid State 61 (2019) 2228-2232, https://doi.org/10.1134/S1063783419110428.

[12] A.Y. Vinogradov, S.A. Grudinkin, N.A. Besedina, S.V. Koniakhin, M.K. Rabchinskii, E.D. Eidelman, V.G. Golubev, Structure and properties of thin graphite-like films produced by magnetron-assisted sputtering, Semiconductors 52 (2018) 914-920, https://doi.org/10.1134/S1063782618070266.

[13] T.A. Friedman, J.P. Sullivan, US Patent 6,103, 305 (2000).

[14] A.C. Ferrari, B. Kleinsorge, N.A. Morrison, A. Hart, Stress reduction and bond stability during thermal annealing of tetrahedral amorphous carbon, J. Appl. Phys. 85 (1999) 7191-7197, https://doi.org/10.1063/1.370531.

[15] A.Ya Kolpakov, A.I. Poplavsky, M.E. Galkina, S.S. Manokhin, J.V. Gerus, The local crystallization in nanoscale diamond-like carbon films during annealing, Appl. Phys. Lett. 105 (2014) 233110, https://doi.org/10.1063/1.4903803.

[16] J.J. Hauser, Electrical, structural and optical properties of amorphous carbon, J. Non-Cryst. Solids 23 (1977) 21-41, https://doi.org/10.1016/0022-3093(77) 90035-7.

[17] N.F. Mott, E.A. Davis, Electronic Processes in Non-crystalline Materials, Oxford university press, 2012.

[18] R. Laiho, K.G. Lisunov, E. Lähderanta, P.A. Petrenko, J. Salminen, M.A. Shakhov, M.O. Safontchik, V.S. Stamov, M.V. Shubnikov, V.S. Zakhvalinskii, Variable-range 
hopping conductivity in $\mathrm{La}_{1-\mathrm{x}} \mathrm{Ca}_{\mathrm{x}} \mathrm{Mn}_{1-\mathrm{y}} \mathrm{Fe}_{\mathrm{y}} \mathrm{O}_{3}$ : evidence of a complex gap in density of states near the Fermi level, J. Phys. Condens. Matter 14 (2002) 8043-8055, https://doi.org/10.1088/0953-8984/14/34/323.

[19] O. Ivanov, M. Yaprintsev, Variable-range hopping conductivity in Lu-doped Bi2Te3, Sol. St. Sci. 76 (2018) 111-117, https://doi.org/10.1016/j. solidstatesciences.2017.12.012

[20] B.I. Shklovskii, A.L. Efros, Electronic Properties of Doped Semiconductors, Springer, 1984

[21] V.I. Ivanov-Omskii, A.V. Tolmatchev, S.G. Yastrebov, Optical properties of amorphous carbon films deposited by magnetron sputtering of graphite, Semiconductors 35 (2001) 220-225, https://doi.org/10.1134/1.1349936.

[22] E.A. Konshina, Absorption and the optical gap of $a-\mathrm{C}: \mathrm{H}$ films produced from acetylene plasmas, Semiconductors 33 (1999) 451-456, https://doi.org/10.1134/ 1.1187897.

[23] J. Robertson, E.P. O'Reilly, Electronic and structure of amorphous carbon, Phys. Rev. B 35 (1987) 2946-2956, https://doi.org/10.1103/PhysRevB.35.2946.

[24] J. Robertson, Electronic and atomic structure of diamond-like carbon, Semicond. Sci. Technol. 18 (2003) S12-S19, https://doi.org/10.1088/0268-1242/18/3/302.

[25] I.A. Faizrakhmanov, V.V. Bazarov, V.A. Zhikharev, I.B. Khaibullin, The effect of bombardment with carbon ions on the nanostructure of diamond-like films, Semiconductors 35 (2001) 591-597, https://doi.org/10.1134/1.1371628.

[26] A.C. Ferrari, S.E. Rodil, J. Robertson, Interpretation of infrared and Raman spectra of amorphous carbon nitrides, Phys. Rev. B 67 (2003) 155306, https://doi.org/ 10.1103/PhysRevB. 67.155306.

[27] A.C. Ferrari, J. Robertson, Raman spectroscopy of amorphous, nanostructured, diamond-like carbon, and nanodiamond, Phil. Trans. R. Soc. Lond. A 362 (2004) 2477-2512, https://doi.org/10.1098/rsta.2004.1452.

[28] S. Neuville, Quantum electronic mechanisms of atomic rearrangements during growth of hard carbon films, Surf. Coat. Technol. 206 (2011) 703-726, https://do org/10.1016/j.surfcoat.2011.07.055.

[29] S. Neuville, Selective carbon material engineering for improved MEMS and NEMS MDPI Micromachines 10 (2019) 539, https://doi.org/10.3390/mi10080539.

[30] A.C. Ferrari, J. Robertson, Interpretation of Raman spectra of disordered and amorphous carbon, Phys. Rev. B 61 (2000) 14095-14107, https://doi.org/ 10.1103/PhysRevB.61.14095.

[31] I.P. Ivanenko, S.V. Krasnoshchekov, A.V. Pavlikov, Analysis of the structure and conductivity of kinked carbon chains obtained by pulsed plasma deposition on various metal substrates, Semiconductors 52 (7) (2018) 907-913, https://doi.org/ 10.1134/S1063782618070102.

[32] L. Ravagnan, F. Siviero, C. Lenardi, P. Piseri, E. Barborini, P. Milani, C.S. Casari, A. Li Bassi, C.E. Bottani, Cluster-beam deposition and in situ characterization of carbyne-rich carbon films, Phys. Rev. Lett. 89 (2002) 285506, https://doi.org/ 10.1103/PhysRevLett.89. 285506.

[33] Bitao Pan, Jun Xiao, Jiling Li, Pu Liu, Chengxin Wang, Guowei Yang, Carbyne with finite length: the one-dimensional sp carbon, Science Advances 1 (2015) 1500857, https://doi.org/10.1126/sciadv.1500857.

[34] Mark D. Tucker, Zsolt Czigány, Esteban Broitman, Lars-Åke Näslund, Lars Hultman, and Johanna Rosen, Filtered pulsed cathodic arc deposition of fullerene- like carbon and carbon nitride films, J. Appl. Phys. 115 (2014) 144312, https:// doi.org/10.1063/1.4871179.

[35] D.B. Williams, C.B. Carter, Transmission Electron Microscope, A Textbook for Materials Science, Springer, 2009.

[36] P.B. Hirsch, A. Howie, R.B. Nicholson, D.W. Pashley, M.J. Whelan (Eds.), Electron Microscopy of Thin Crystals, Butterworths, London, 1965.

[37] S. Bhattacharyya, M. Lübbe, P.R. Bressler, D.R.T. Zahn, F. Richter, Structure of nitrogenated amorphous carbon films from NEXAFS, Diamond Relat. Mater. 11 (2002) 8-15, https://doi.org/10.1016/S0925-9635(01)00525-8.

[38] S.S. Roy, R. McCann, P. Papakonstantinou, P. Maguire, J.A. McLaughlin, The structure of amorphous carbon nitride films using a combined study of NEXAFS, XPS and Raman spectroscopies, Thin Solid Films 482 (2005) 145-150, https://doi. org/10.1016/j.tsf.2004.11.132.

[39] Niklas Hellgren, Jinghua Guo, Yi Luo, Conny Såthe, Akane Agui, Stepan Kashtanov, Nordgren Joseph, Hans Ågren, Jan-Eric Sundgren, Electronic structure of carbon nitride thin films studied by X-ray spectroscopy techniques, Thin Solid Films 471 (2005) 19-34, https://doi.org/10.1016/j.tsf.2004.03.027.

[40] Ganjigunte R.S. Iyer, Paul D. Maguire, Metal free, end-opened, selective nitrogendoped vertically aligned carbon nanotubes by a single step in situ low energy plasma process, J. Mater. Chem. 21 (2011) 16162-16169, https://doi.org/ 10.1039/C1JM12829A.

[41] F. Alvarez, M.C. dos Santos, Electronic and structural properties of amorphous carbon-nitrogen alloys, J. Non-Cryst. Solids 266-269 (2000) 808-814, https://doi. org/10.1016/S0022-3093(99)00820-0.

[42] Xinyi Zhang, Reinhard Schneider, Erich Müller, Dagmar Gerthsen, Practical aspects of the quantification of $\mathrm{sp}^{2}$-hybridized carbon atoms in diamond-like carbon by electron energy loss spectroscopy, Carbon 102 (2016) 198-207, https://doi.org/ 10.1016/j.carbon.2016.02.020.

[43] E.D. Eidelman, On a carbon nanostructure-based thermoelectric converter with record parameters, Semiconductors 51 (2017) 906-908, https://doi.org/10.1134/ S1063782617070089.

[44] Y. Iijima, T. Harigai, R. Isono, S. Degai, T. Tanimoto, Y. Suda, H. Takikawa, H. Yasui, S. Kaneko, S. Kunitsugu, M. Kamiya, M. Taki, Preparation of multi-layer film consisting of hydrogen-free DLC and nitrogen-containing DLC for conductive hard coating, in: AIP Conference Proceedings, 1929, 020024, https://doi.org/ 10.1063/1.5021937, 2018.

[45] M. B Taylor, D.W.M. Lau, J.G. Partridge, D.G. McCulloch, N.A. Marks, E.H.T. Teo, D.R. McKenzie, The origin of preferred orientation during carbon film growth, J. Phys. Condens. Matter 21 (2009) 225003, https://doi.org/10.1088/0953-8984/ $21 / 22 / 225003$

[46] M. Shakerzadeh, M. Parrot, Y. Man, Y. Yang, H. Xie, Wettability, nanoscratch resistance and thermal stability of filtered cathodic vacuum arc grown nitrogenated amorphous carbon films, Surf. Coat. Technol. 292 (2016) 30-36, https://doi.org/10.1016/j.surfcoat. 2016.03.018.

[47] E. Broitman, N. Hellgren, K. Järrendahl, M.P. Johansson, S. Olafsson, G. Radnóczi, J.-E. Sundgren, L. Hultman, Electrical and optical properties of CNx $(0 \leq x \leq 0.25)$ films deposited by reactive magnetron sputtering, J. Appl. Phys. 89 (2001) 1184-1190, https://doi.org/10.1063/1.1334370. 